\title{
Impact of the Synthesis Method on the Solid-State Charge Transport of Radical Polymers
}

\author{
Yiren Zhang $^{\mathrm{a}, \dagger}$, Albert Park ${ }^{\mathrm{b}, \dagger}$, Alicia Cintora ${ }^{\mathrm{a}}$, Stephen R. McMillan ${ }^{\mathrm{c}}$, Nicholas J. Harmon ${ }^{\mathrm{c}}$, \\ Austin Moehle $^{\mathrm{b}}$, Michael E. Flattéc ${ }^{\text {, Gregory D. Fuchs }}{ }^{\mathrm{b}}$, and Christopher K. Ober ${ }^{\mathrm{a}}$ \\ aMaterials Science and Engineering, Cornell University, Ithaca, New York 14853, USA \\ ${ }^{b}$ Applied and Engineering Physics, Cornell University, Ithaca, New York 14853, USA \\ 'Optical Science and Technology Center and Department of Physics and Astronomy, University of \\ lowa, lowa City, lowa 52242, USA
}

\begin{abstract}
There are conflicting reports in the literature about the presence of room temperature conductivity in poly(2,2,6,6-tetramethylpiperidinyloxy methacrylate) (PTMA), a redox active polymer with radical groups pendent to an insulating backbone. To understand the variability in the findings across the literature and synthetic methods, we prepared PTMA using three living methods anionic, ATRP and RAFT polymerization. We find that all three synthetic methods produce PTMA with radical yields of $70-80 \%$, controlled molecular weight, and low dispersity. Additionally, we used on-chip EPR to probe the robustness of radical content in solid films under ambient air and light, and found negligible change in the radical content over time. Electrically, we found that PTMA is highly insulating - conductivity in the range $10-{ }^{11} \mathrm{~S} / \mathrm{cm}$ - regardless of the synthetic method of preparation. These findings provide greater clarity for potential applications of PTMA in energy storage.
\end{abstract}

\section{Graphical abstract}

Though poly(2,2,6,6-tetramethylpiperidinyloxy methacrylate) (PTMA) was reported to be conductive, careful measurements indicate its insulating nature regardless of synthesis method, which have implication for its application in organic electronics.

\footnotetext{
$\dagger$ These authors contributed equally to this work.

Electronic Supplementary Information (ESI) available: See DOI: 10.1039/x0xx00000x

Conflicts of interest

There are no conflicts to declare.
} 

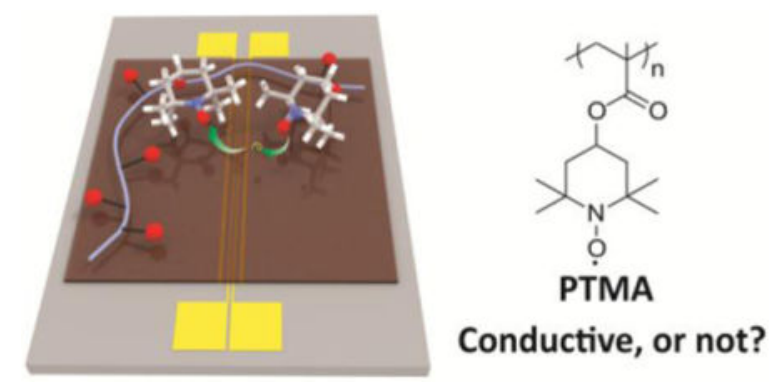

\section{Introduction}

Radical polymers, a class of polymers with robust radical pendent moieties along their backbone, have demonstrated suitable performance to meet the requirements for energy storage applications such as organic radical batteries (ORBs). ${ }^{1-6}$ Poly $(2,2,6,6-$ tetramethylpiperidinyloxy methacrylate) (PTMA) remains the most popular stable radical polymer for ORBs due to its facile preparation and fast redox kinetics of the 2,2,6,6tetramethyl pipiridine-1-oxyl (TEMPO) stable radical moiety side group. The incorporation of TEMPO moieties into polymers also has a rich history in the study of such diverse applications as organic magnets ${ }^{7}$ and is currently being explored in antifouling coatings as a component to inhibit curing of biological adhesives ${ }^{8}$. Such structures are also associated with certain types of stable radical polymerization. ${ }^{9}$

Pristine PTMA has been largely considered to be an electrically insulating material in ORBs, which has been synthesized primarily via radical polymerization and subsequent oxidation. ${ }^{10-13}$ As a result, many attempts have been made to improve its charge transport capabilities in battery electrodes, such as by incorporating carbon black-based additives to the active cathode material. ${ }^{14-16}$ Recently, however, it has been reported that thin films of PTMA synthesized via reversible addition-fragmentation chain transfer (RAFT) can be considered as a novel class of highly-transparent polymers with a conductivity of $\sim 1 \times 10^{-6} \mathrm{~S} / \mathrm{cm}$, comparable to $\pi$-conjugated semiconducting polymers such as undoped poly(3hexylthiophene) (P3HT). ${ }^{17-19}$ Motivated by the lack of agreement over the intrinsic conductivity of PTMA, we have set out to understand the impact of different synthetic preparation routes on charge transport.

Despite the versatility of synthetic techniques, the only prior reported preparation of conductive PTMA homopolymer follows a two-step process which involves the preparation of a precursor polymer, poly(2,2,6,6-tetramethyl-4-piperidyl methacrylate) (PTMPM) by RAFT and subsequent oxidation by peracid. ${ }^{17,20}$ Limited by the specific chemistry of polymerization or chemical treatment, the resulting PTMA is often accompanied by unwanted functionalities due to incomplete conversion or side reactions. These additional functionalities were found to have an effect on the measured solid state conductivity of PTMA thin films. ${ }^{20}$ Efforts to understand the charge transport in these systems found that the intrinsic conductivity of PTMA can be increased by a factor of two by incorporating the oxoammonium cation, which can be introduced either by chemical oxidation of PTMPM or 
as an external dopant. The conductivity enhancement was attributed to the oxoammonium cations serving as additional hopping sites for the nitroxide radical. ${ }^{20,21}$

In support of these results, charge transport in electrochemical systems has also been considered in the literature. ${ }^{22}$ TEMPO stable radicals pendent to an insulating, hydrocarbon backbone can effectively transport charge with the aid of an electrolyte solution. It has been suggested that in these systems, charge carriers travel from TEMPO group to TEMPO group via a variable range hopping mechanism enabled by chain motion. ${ }^{5,23,24}$ This model suggests that there can be a series of electron hops in the polymer from pendent site to pendent site with the aid of delocalized electrons. As such, elucidating the nature of the electron transport process in non-conjugated macromolecules with pendent stable radical groups could aid in opening new pathways to the designs of organic electronic materials.

Given the disagreement on the conductivity of non-conjugated stable radical polymers, direct comparison of PTMA prepared by different synthesis techniques is a crucial test of the robustness of charge transport phenomena that can reveal the role of structure and impurities in conductivity.

In this work, we have created a set of PTMA polymers synthesized via three different synthetic methods to understand how the variation of resulting pendent groups can affect the intrinsic conductivity of PTMA thin films. We used two living radical polymerization methods, RAFT and atom transfer radical polymerization (ATRP), for the preparation of two precursor polymers of PTMPM, which were then transformed to PTMA polymers (PTMARAFT, PTMA- $\mathrm{H}_{2} \mathrm{O}_{2}$, PTMA- $m$ CPBA) by oxidation reactions. ATRP of 4methacryloyloxy-1-((1'-phenylethyl)oxy-2,2,6,6-tetramethylpiperidine (MPEOT) resulted in the precursor polymer PMPEOT, which was transformed to PTMA- $\mathrm{O}_{2}$ by oxidative C-O bond cleavage of the polyalkoxyamines ${ }^{25}$ (Scheme 1). For comparison, we also produced PTMA (PTMA-Anionic) with both low molecular weight distribution and high TEMPO stable radical yield via anionic polymerization. Considering that this method can directly polymerize the TEMPO-bearing methacrylate monomer with few side reactions, PTMAAnionic can act as a suitable model polymer for physical characterization.

To characterize conductivity, we used equipment designed for measuring high-resistance PTMA samples in a 4-wire geometry. This both avoids the effect of contact resistance and eliminates artifacts that can occur when the sample resistance is higher than the meter input impedance. In the following sections, we describe our studies on this comprehensive measurement of PTMA conductivity, which is a key step to the understanding of charge transport in a neat PTMA film. Finally, we analyse the results in the context of a variable range hopping theoretical analysis.

\section{Experimental section}

\subsection{Materials}

All chemicals were purchased from Sigma-Aldrich and used as received unless otherwise noted. $m$-Chloroperbenzoic acid ( $m$ CPBA) was washed with DI water from diethyl ether and dried under vacuum. 2,2,6,6-Tetramethyl-4-piperidyl methacrylate (TMPM) and dibenzo-18- 
crown-6 were purchased from TCI America. Dibenzo-18-crown-6 was recrystallized from acetonitrile and dried under vacuum overnight. $\mathrm{CuBr}$ was stirred in acetic acid for overnight, washed repeatedly with anhydrous ethanol and diethyl ether and dried under vacuum before use. The TEMPO methacrylate monomer and 2-phenyl-2-propylbenzodithioate ${ }^{26}$ were synthesized according to literature procedures.

\subsection{Characterization}

Molecular weights $\left(\mathrm{M}_{\mathrm{n}}\right)$ and polydispersities $\left(\mathrm{M}_{\mathrm{w}} / \mathrm{M}_{\mathrm{n}}\right)$ of the PTMA samples and precursor polymers were dertermined by gel permeation chromatography (GPC). The GPC was performed on Waters Ambient Temperature GPC equipped with a Waters 410 differential refractive index detector. GPC columns were eluted at $1.0 \mathrm{~mL} / \mathrm{min}$ with tetrahydrofuran (THF) at $40{ }^{\circ} \mathrm{C}$. Polymer samples were dissolved in THF at $1 \mathrm{mg} / \mathrm{mL}$. Polystyrene standards with narrow molecular weight distribution were used for calibration. ${ }^{1} \mathrm{H}-\mathrm{NMR}$ spectra were recorded on a Varian INOVA $400\left({ }^{1} \mathrm{H}, 400 \mathrm{MHz}\right)$ spectrometer. Spectra were referenced to the residual chloroform $(7.26 \mathrm{ppm})$ signal. The ATR-FTIR spectra ranging between wavenumbers 4000 and $400 \mathrm{~cm}^{-1}$ were recorded under vacuum with Bruker Vertex V80V Vacuum FTIR system. Spectra resolution was set at $4 \mathrm{~cm}^{-1}$.

\subsection{Polymer synthesis}

2.3.1 PTMA-anionic synthesis-Dibenzo-18-crown-6 (0.126 g, $0.35 \mathrm{mmol})$ and a stir bar were placed in a hot Schlenk flask with a Rotaflo stopcock and was taken into a nitrogen filled glovebox, where $50 \mathrm{~mL}$ of THF were added. The flask was sealed with a septum and transferred to an acetone/dry ice bath at $-78^{\circ} \mathrm{C}$ where the flask was purged with argon for $20 \mathrm{~min}$. All consecutive additions were measured in the nitrogen glovebox and added via syringe. Approximately $1 \mathrm{~mL}$ of a $1.4 \mathrm{M}$ solution of sec-butyllithium solution in cyclohexane was added to the flask in order to react with any additional impurities. The flask was then allowed to warm up to room temperature for $1 \mathrm{~h}$. After cooling to $-78{ }^{\circ} \mathrm{C}$ for an additional $20 \mathrm{~min}, 25 \mu \mathrm{L}(0.035 \mathrm{mmol})$ of sec-butyllithium and $25 \mu \mathrm{L}(0.14 \mathrm{mmol})$ of diphenylethylene (DPE) were added and the mixture turned bright red, indicating a successful reaction. After stirring for $30 \mathrm{~min}, 87.5 \mu \mathrm{L}(0.175 \mathrm{mmol})$ of a $2.0 \mathrm{M}$ sodium tertbutoxide solution in THF were added, and there was no color change. After stirring for 45 min, a TEMPO methacrylate solution in THF was slowly added, and allowed to react for 5 $\mathrm{h}$. The reaction was quenched with $1 \mathrm{~mL}$ of degassed methanol, and allowed to cool to room temperature. The THF was removed by rotavap, and the polymer residue was re-dissolved in chloroform. Once re-dissolved, 3 consecutive water washes were done to remove the excess sodium in the solution. The washed polymer/chloroform solution was precipitated in cold hexanes, and dried under vacuum overnight.

2.3.2 PMPEOT synthesis-MPEOT ( $345 \mathrm{mg}, 1.00 \mathrm{mmol}, 1.00$ equiv) was placed in a Schlenk tube under nitrogen. Then $1 \mathrm{~mL}$ of a solution of Ethyl 2-bromo-2-methylpropionate (2-EBiB) $(15 \mu \mathrm{L}, 100 \mathrm{~mol})$ and 1,1,4,7,7-pentamethyldiethylenetriamine (PMDETA) $(21 \mu \mathrm{L}$, $100 \mu \mathrm{mol})$ in anisole $(10 \mathrm{~mL})$ was added. The tube was subjected to three freeze-pump-thaw cycles, and the mixture was transferred via a canular to a Schlenk tube with $\mathrm{CuBr}(1.4 \mathrm{mg}$, $10 \mu \mathrm{mol}, 1.0 \mathrm{~mol} \%$ ) was added. The mixture was stirred at $50{ }^{\circ} \mathrm{C}$ for $9 \mathrm{~h}$. The crude product was precipitated in $25 \mathrm{~mL}$ of cold methanol. The precipitate was collected and washed with 
methanol. It was dissolved in a small amount of $\mathrm{CH}_{2} \mathrm{Cl}_{2}$, and filtered through an aluminum oxide column. The filtered solution was concentrated under vacuum, and precipitated in cold methanol. The polymer was collected and dried under vacuum at $40{ }^{\circ} \mathrm{C}$ overnight.

2.3.3 PTMPM oxidation with mCPBA-A solution of mCPBA (480 mg, $2.78 \mathrm{mmol}, 2.6$ eq.) in $4.8 \mathrm{~mL}$ of anhydrous $\mathrm{CH}_{2} \mathrm{Cl}_{2}$ was made. PTMPM $(0.24 \mathrm{~g}, 1.07 \mathrm{mmol}$ of amine functions, 1 eq.) was dissolved in $4.8 \mathrm{~mL}$ of anhydrous $\mathrm{CH}_{2} \mathrm{Cl}_{2}$ at $0{ }^{\circ} \mathrm{C}$. The $\mathrm{mCPBA}$ solution was added dropwise to the polymer solution and allowed to stir at room temperature for $3 \mathrm{~h}$. After completion, the mixture was extracted with $\mathrm{CH}_{2} \mathrm{Cl}_{2}$ and washed repeatedly with sodium carbonate solution. The organic phase was dried with anhydrous $\mathrm{MgSO}_{4}$, concentrated under vacuum and precipitated in hexane. The polymer was collected and dried under vacuum at $40{ }^{\circ} \mathrm{C}$ overnight.

2.3.4 PTMPM oxidation with Na2WO4/Na2EDTA/H2O2—PTMPM (232 mg, 1.03 mmol of amine functions, 1 eq.), $\mathrm{Na}_{2} \mathrm{WO}_{4} \cdot 2 \mathrm{H}_{2} \mathrm{O}\left(85 \mathrm{mg}, 0.26 \mathrm{mmol}, 0.25\right.$ eq.). $\mathrm{Na}_{2} \mathrm{EDTA}$. $2 \mathrm{H}_{2} \mathrm{O}(45 \mathrm{mg}, 0.15 \mathrm{mmol}, 0.15 \mathrm{eq})$ and methanol $(12 \mathrm{~mL})$ was added to a three-neck roundbottom flask with a condenser. The solution was stirred at $60{ }^{\circ} \mathrm{C}$ for 5 min and $\mathrm{H}_{2} \mathrm{O}_{2}(1.17$ $\mathrm{mL}, 10.3 \mathrm{mmol}, 10$ eq.) was added dropwise over $60 \mathrm{~min}$. The mixture was further stirred at $60{ }^{\circ} \mathrm{C}$ for $24 \mathrm{~h}$. The polymer was extracted with $\mathrm{CH}_{2} \mathrm{Cl}_{2}$, washed repeatedly with water and dried with anhydrous $\mathrm{MgSO}_{4}$. After filtration, the solution was concentrated and precipitated in cold hexane, filtered and dried under vacuum at $40{ }^{\circ} \mathrm{C}$ overnight.

2.3.5 PMPEOT Oxidation-100 mg PMPEOT was dissolved in $10 \mathrm{~mL}$ of tertbutylbenzene $(\mathrm{c}=10 \mathrm{mg} / \mathrm{mL})$, and the mixture was heated at $135^{\circ} \mathrm{C}$ for $5 \mathrm{~h}$ with a reflux condenser. During the reaction dry oxygen (produced by $\mathrm{MnO}_{2}$ catalyzed $\mathrm{H}_{2} \mathrm{O}_{2}$ decomposition, dried over anhydrous $\mathrm{CaCl}_{2}$ ) was bubbled through the solution. After completion, tert-butylbenzene was removed under vacuum, and the solid was dissolved in a minimum amount of $\mathrm{CH}_{2} \mathrm{Cl}_{2}$. The polymer was collected from precipitation in cold hexane and dried under vacuum at $40{ }^{\circ} \mathrm{C}$ overnight.

\subsection{Solution-state spin characterization}

Solution-state measurements were performed in a Bruker X-band EPR spectrometer at room temperature. PTMA solutions for EPR measurements (1 mM of repeating unit concentration) were prepared by dilution of $384.6 \mu \mathrm{L}$ of PTMA stock solutions to $4.00 \mathrm{~mL}$ of total volume with toluene. Stock solution was prepared by dissolving $10.0 \mathrm{mg}$ of each polymer in $4.00 \mathrm{~mL}$ toluene overnight. The resulting EPR spectra were double-integrated and compared with the TEMPO standard (1 mM solution in toluene) to calculate radical yields. Error bars were generated by calculating the standard deviation of average radical yield from three parallel quantitative EPR tests in which the same PTMA stock solution was used to prepare independent test solutions.

\subsection{Solid-state spin characterization using a coplanar waveguide}

Samples were prepared either by drop casting or spin coating a $10 \mathrm{mg} / \mathrm{mL}$ solution of PTMA directly onto the microfabricated coplanar waveguide meander. ${ }^{27}$ The waveguide was then wire-bonded into the microwave chip carrier, and the $6 \mathrm{GHz}$ transmitted microwave power 
was monitored as a function of magnetic field with a microwave diode and a lock-in amplifier. Error bars were calculated by propagating errors including the uncertainties from the fitting and the noise in the lock-in voltage.

\subsection{Electrical characterization}

Micro-fabricated 4-wire lateral templates consisting of 100-nm-thick, 2.5-mm-long gold electrodes were patterned on insulating, thermally oxidized Si substrates. The four electrodes are equally spaced at a distance of either 2, 5, 10, or $20 \mu \mathrm{m}$ (Fig. 1). We used more than one electrode spacing for each PTMA type to validate that the results are not an artifact of the geometry. PTMA films were prepared by dropcasting PTMA solution (25 $\mathrm{mg} / \mathrm{mL}$ in toluene) on a template, and heated at $70{ }^{\circ} \mathrm{C}$ for $15 \mathrm{~min}$ to remove the residual toluene. PTMA film thickness was measured using both an ellipsometer and Tencor AlphaStep 500 profilometer. After wire-bonding the template into a chip carrier, each sample was placed within a continuously pumped vacuum box to eliminate resistive shunting through a surface water layer. Current was applied to the outer electrodes and measure voltage between the inner electrodes. To eliminate a systematic error that can arise when measuring samples with very large resistance (a typical resistance is between 60 to $200 \mathrm{G} \Omega$ in our experiment) relative to the input impedance of a standard voltmeter, a Keithley 6514 electrometer that has an input impedance of $210 \mathrm{~T} \Omega$ was used. Current was sourced using a Keithley 6610 precision current source enabling current with sub-1 pA accuracy. The set-up was calibrated against standard Ohmite MOX resistors in the range 100 $\mathrm{G} \Omega$ to $1 \mathrm{~T} \Omega$. (for the details of calibration please refer to the supplemental information) As a control, the resistance of a 4-wire template in our set-up without a PTMA film under the same conditions was measured and found that the resistance was above $10 \mathrm{~T} \Omega$ which exceeds the reasonable range for calibration. All measurements were done in a Faraday cage to prevent electrostatic artifacts.

\section{Results and discussion}

\subsection{Polymerization}

Scheme 1 summarizes our synthetic strategy of PTMA synthesis in this study. In previous reports, PTMA synthetic routes mainly fall into two categories: (i) direct polymerization of TEMPO-containing methacrylate monomer using reactions that are compatible with stable radicals, such as anionic polymerization ${ }^{28}$ and group-transfer polymerization ${ }^{29}$; and (ii) synthesis of precursor polymers via radical polymerization, which can be terminated by nitroxide radical, and subsequent radical regeneration via chemical treatment. To overcome the intrinsic drawback of traditional radical polymerizations, including lack of control over the molar mass and the dispersity, reversible-deactivation polymerizations allow the creation of well controlled PTMA precursor polymer due to their fast initiation rates and consistent concentration of active sites. To minimize the influence of dispersity, here we used only living polymerization, including anionic polymerization, ATRP and RAFT.

Table 1 summarizes the corresponding polymerization methods, chemical oxidation, molecular weights and dispersity index (PDI) of synthesized PTMA samples. All of the PTMA products show low PDI of $1.15-1.28$, which is in agreement with previous reports. 
Among them, PTMA-anionic show a narrow dispersity, indicating that the TEMPO bearing methacrylate monomers can be polymerized with high suppression of side reactions on nitroxide radical via sodium-mediated anionic polymerization. Such controlled polymer structures are desired in exploration of the effects of different existing pendent groups due to minimization of molecular weight and distribution effect.

\subsection{Oxidation}

Due to the fact that modern synthetic chemistry of stable nitroxides developed historically from piperidine derivatives, the oxidation of sterically hindered secondary amines by peroxides and peracids has been the most popular preparative method for the synthesis of PTMA. Though various reaction conditions have been used, including $\mathrm{H}_{2} \mathrm{O}_{2} /$ EDTA/ $\mathrm{Na}_{2} \mathrm{WO}_{4}$ combination and $m$ CPBA, the oxidation of amine actually occurs by a similar mechanism. ${ }^{30}$ In the first stage, the hydroxylamino derivative $(\mathrm{N}-\mathrm{OH})$ is generated as a major product, which is converted into an oxoammonium moiety $\left({ }^{+} \mathrm{N}=\mathrm{O}\right)$ via subsequent oxidation. The consumption of ${ }^{+} \mathrm{N}=\mathrm{O}$, the intermediate product, is realized by reaction with reducing species, which is hydroxylamino derivative (N-OH) for $m C P B A$ system and $\mathrm{H}_{2} \mathrm{O}_{2}$ derivative for $\mathrm{H}_{2} \mathrm{O}_{2} / \mathrm{EDTA} / \mathrm{Na}_{2} \mathrm{WO}_{4}$ system.

Meanwhile, thermolysis of alkoxyamine under aerated conditions can also be a reliable method for efficient TEMPO recovery, considering its simpler mechanism and less intermediate species compared with the oxidation of amines. Here, optimized oxidation conditions using either $\mathrm{H}_{2} \mathrm{O}_{2} / \mathrm{Na}_{2} \mathrm{EDTA} / \mathrm{Na}_{2} \mathrm{WO}_{4}$ combination and $m C P B A$ were adopted for our PTMPM samples, while PMPEOT polymer were deprotected in tert-butylbenzene at $135{ }^{\circ} \mathrm{C}$ under oxygen atmosphere. After oxidation, all the PTMA samples were pale orange solid, while corresponding polymer solutions exhibited the characteristic absorption of TEMPO moiety at $463 \mathrm{~nm}$, suggesting the successful generation of TEMPO radical in all the PTMA samples.

Prevention of unintentional oxoammonium $\left({ }^{+} \mathrm{N}=\mathrm{O}\right)$ production is important to our study, since we want to establish the base conductivity of pristine PTMA, and oxoammonium has been reported previously as a conductivity enhancing dopant. ${ }^{21,22,31,32}$ We used attenuated total internal reflectance-Fourier transform infrared (ATR-FTIR) spectroscopy to study the existence of ${ }^{+} \mathrm{N}=\mathrm{O}$ species in our PTMA samples as shown in Figure 2. Compared with their precursor polymers, all the PTMA samples exhibit the characteristic peak of $\mathrm{N}-\mathrm{O} \bullet$ at 1365 $\mathrm{cm}^{-1}$, while only PTMA oxidized by mCPBA show an observable single peak at $1540 \mathrm{~cm}^{-1}$, which corresponds to the ${ }^{+} \mathrm{N}=\mathrm{O}$. Beside the disproportionation and over-oxidation by $m C P B A$, this can also be explained by the inefficient reduction of intermediate ${ }^{+} \mathrm{N}=\mathrm{O}$ formed in amine oxidation by polymer-bounded $\mathrm{N}-\mathrm{OH}$, while reduction by excess $\mathrm{H}_{2} \mathrm{O}_{2}$ in PTMA- $\mathrm{H}_{2} \mathrm{O}_{2}$ is enough for complete ${ }^{+} \mathrm{N}=\mathrm{O}$ reduction. The unavoidable presence of ${ }^{+} \mathrm{N}=\mathrm{O}$ is consistent with lower radical conversion in PTMA oxidized by $m$ CPBA as compared with other methods. In the samples not oxidized with $m \mathrm{CPBA}$, we conclude that the pendent groups consist only of TEMPO stable radicals and a small fraction of corresponding precursor groups. These materials with their high purity and range of synthetic strategies appear to be suitable systems for the subsequent conductivity tests. 


\subsection{Spin characterization}

We measured radical yields using quantitative solution-state electron paramagnetic resonance (EPR). Figure 3(b) shows that the radical yield of PTMA, regardless of synthesis approach, varied by only 10 percent within the range 70 to $80 \%$. PTMA-Anionic had the highest average yield of 77\%, while PTMA-ATRP showed lowest yield of 70\%. Although we prepared PTMA using different polymerization and oxidation methods, we found no significant variation in radical content among all PTMA samples (Figure 3(b)). The absence of a triplet signal, which originates either from free-floating TEMPO molecules or isolated radical groups along the polymer chain, further confirms high radical contents and good purity in our PTMA samples. Consistent with FTIR results and with our understanding of the synthetic methods, we expect that most of side-groups that are not converted to radicals are not electrically active oxoammonium.

Motivated to understand the stability of the radical spins in solid films, which is relevant for applications, we also performed room temperature EPR of solid PTMA films using a broadband, on-chip coplanar waveguide set-up shown in Fig. 4a-b. The transmitted microwave power, $P$, that passes through a meandering coplanar waveguide is demodulated with respect to a $6 \mathrm{G}, 587 \mathrm{~Hz}$ magnetic field, $H$, modulation. This is a measurement of $\mathrm{d} P / \mathrm{d} H$ which rejects $H$ independent microwave power transmission. As the static component of $H$ is swept through the spin resonance, we expect a single, derivative-of-Lorentzian lineshape because of the high radical yield in our materials and because of the decreased radical inter-chain spacing within a solid film. We verified that the signal scales with film thickness as expected for thin films, and that spin-coating and drop-casting give similar amplitudes once normalized for film thickness.

The robustness of radical spins in PTMA exposed to ambient light and air is a particular concern for many applications, including for conductive polymer materials. ${ }^{33-36}$ Although the radical spins in PTMA films are generally believed to be stable, there has been no prior quantitative test in solid films, which is the relevant form of the material for applications. Figure 4(c) shows our measurement of waveguide EPR, which is representative of all of our measurements of PTMA materials in this study. Because we measure the solid-state form of PTMA, the singlet linewidth is larger than that measured in solution state due to a closer spacing between radical groups, which enhances exchange narrowing. ${ }^{37,38}$ Without breaking any microwave contacts, we repeated our measurement of the solid-state EPR over a period of eight days (Figs. 4d-e), over which the sample was exposed to ambient fluorescent lighting and air. We found a negligible decrease in the EPR signal amplitude over that period. We also re-measured PTMA samples after 5 months without a significant change to the EPR amplitude. However, we note that there are small variations in signal amplitudes that originate from breaking and re-making microwave contacts that adds uncertainty to quantitative comparisons. From these measurements, we conclude that radical groups in PTMA solid films are robust to ambient conditions.

\subsection{Conductivity measurement}

We performed electrical measurements of solid PTMA films to understand how different synthetic methods influence the steady-state electrical conductivity of this material. We 
chose a lateral, 4-wire geometry in this measurement for two reasons: first, it eliminates contact resistance, which may vary significantly depending on the contact electrode materials and surface preparation. Second, it eliminates the potential for unintentional parallel conductance pathways that could occur in a vertically stacked sandwich structure $^{18-21,32}$ (e.g. bottom electrode, PTMA film, top electrode deposited on top via evaporation). For example, these structures are susceptible to pin-holes and uncontrolled diffusion of the top electrode into the polymer film, which provide a shunt resistive path. Furthermore, because the polymer film is not over-coated in metal, residual solvent could persist in the polymer if it is not fully baked out after PTMA film deposition. Finally, we note that unintentional conductivity is difficult to check for in vertically-stacked structures. Thus, we choose to eliminate these uncertainties by working in a lateral measurement geometry, consistent with some prior studies. ${ }^{17}$

Table 2 summarizes our findings for PTMA-Anionic, PTMA- $\mathrm{H}_{2} \mathrm{O}_{2}$ and PTMA-RAFT. Note that of the three ATRP-synthesized PTMA materials, we studied only PTMA- $\mathrm{H}_{2} \mathrm{O}_{2}$ electrically because it had the largest radical yield of that group. We found that while the conductivity of PTMA prepared using different synthetic methods varies by a factor of $\sim 5$, all samples were highly insulating, independent of synthetic approach. The conductivity values that we report, between $1.09 \pm 0.76 \times 10^{-11}$ and $5.59 \pm 0.78 \times 10^{-11} \mathrm{~S} / \mathrm{cm}$, are orders of magnitude smaller than some prior reports ${ }^{18-21}$ but in agreement with others. ${ }^{32}$ This could indicate that while ionic transport between TEMPO moieties can lead to much higher conductivity as compared to PMMA, which has conductivity in range of $10^{-14} \sim 10^{-15} \mathrm{~S} / \mathrm{cm}$, it is far below the conductivity of organic semiconductors with a $\pi$-conjugated backbone. For confirmation of this statement, we also measured the resistance of an undoped regioregular P3HT film, which is a common conjugated polymer. We found a conductivity of $4.5 \times 10^{-8} \mathrm{~S} / \mathrm{cm}$, which is 2 orders of magnitude smaller than typically reported ${ }^{39}$ but which is consistent with the lack of crystallinity from drop casting and with normal sample aging. ${ }^{40}$ Nonetheless, we found that the P3HT film is 3 orders of magnitude more conductive than PTMA films, regardless of synthetic preparation.

To understand these results in the context of radical-mediated electronic transport mechanisms, here we theoretically estimate the number of radical sites involved in electronic transport. We consider charge transport due to phonon-assisted tunnelling between sites with Mott variable range hopping, ${ }^{41}$ which assigns hopping rates depending on both the wave function overlap and also any thermal activation required to hop. An increase in site separation, or a difference in site energy, both lead to lower hopping rates ${ }^{42}$ by reducing the density of states $g(\mu)$, near the Fermi level $\mu$. The concentration of conducting sites correspond to $N_{\mathrm{c}}=2 g(\mu) \varepsilon_{0}^{\mathrm{opt}}$, where the optimal bandwidth

$$
\varepsilon_{0}^{\mathrm{opt}}=\left(\frac{k_{\mathrm{B}} T}{a}\right)^{1 / 3}\left(\frac{1}{g(\mu)}\right)^{1 / 4}
$$

where $a$ is the carrier's wave function localization length. From these assumptions, the resistivity in an amorphous semiconducting material is expressed as 


$$
\rho=\rho_{0} \operatorname{Exp}\left[\left(\frac{T_{0}}{T}\right)^{1 / 4}\right]
$$

where $T_{0}=\beta /\left[a^{3} k_{\mathrm{B}} g(\mu)\right]$ with $\beta$ a percolative constant, ${ }^{41}$ and $\rho_{0}$. a material dependent constant describing the minimum resistivity based on carrier-phonon interactions, ${ }^{41}$

$$
\rho_{0}=\frac{9 \pi}{4} \frac{a^{3} d s^{5} \hbar^{4} \kappa^{2}}{E_{1}^{2} e^{6} k_{\mathrm{c}}^{2}}\left(\frac{\beta}{a^{3} g(\mu) k_{\mathrm{B}} T}\right)^{(\nu+2) / 4}
$$

In the above expression $d$ is the mass density of the dielectric, $s$ is the speed of sound in the dielectric, ${ }^{43} \kappa$ is the dielectric constant, ${ }^{44} E_{1}$ is the deformation potential, ${ }^{45} e$ is the electronic charge, $k_{\mathrm{c}}$ is the Coulomb constant, and $\nu$ is a numerical constant determined through percolation simulations. ${ }^{41}$ An estimate of the density of states is made by equating Eq (2) with measured values of the resistivity (see supplement). For the anionic PTMA sample with $\rho=9.19 \times 10^{10} ; \Omega \cdot \mathrm{cm}, g(\mu)=2.5 \times 10^{20} \mathrm{eV}^{-1} \cdot \mathrm{cm}^{-3}$, and from Eq (1), $\varepsilon_{0}^{\mathrm{opt}}=0.52 \mathrm{eV}$. It is assumed that each monomer of PTMA contains one side chain and given a mass density of $\mu_{\mathrm{s}}=1.018 \mathrm{~g} \cdot \mathrm{cm}^{-3}$ and the molecular weight of a monomer $w=240.32 \mathrm{Da}$, the average spacing between sites is $r=4.5 ; \AA \AA \AA$. The concentration of conducting sites is $N_{\mathrm{c}}=2.5 \times 10^{20} \mathrm{~cm}^{-3}$ and so the ratio of conducting sites to insulating sites, $f$, is

$$
f=N_{\mathrm{c}} \frac{4 \pi}{3} r^{3}
$$

For the parameters outlined above, $f=0.1$ suggesting that about $10 \%$ of sites within the sample contribute to charge transport, with on average half of these sites occupied and half empty of carriers - a statement of single occupancy. This corresponds to approximately $5 \%$ of sites being unoccupied and able to host a carrier, which is consistent with our FTIR measurements and our expectation that nearly all side groups that are not converted to radical sites are not electrically active.

\section{Conclusions}

In this study, we have prepared a series of PTMA materials of controlled molecular weight and dispersity using a range of living polymerization methods. We have used both controlled radical and anionic polymerizations to make the target polymers. Anionic polymerization enables formation of narrow dispersity polymer directly from the stable radical monomer. Controlled radical methods require that we polymerize from a precursor amine to make a polymer and then oxidize the polymer to form the stable radical. Using solution EPR methods to evaluate the stable radical content in each polymer, we find that all synthetic methods result in a TEMPO content per repeat of between 70 and 80 percent. Only in the case of peracid oxidation can we observe by IR spectroscopy an overoxidation to form an 
oxoammonium species. We also used an on-chip EPR technique to probe the stability of solid PTMA films, finding no appreciable change in the radical content for more than a week. Having established the high-quality synthesis of stable radical polymers, we then investigate the electrical conductivity in these materials using lateral template devices that enable a four-point measurement. We find that independent of synthetic method, these polymers are highly insulating, with conductivities ranging from $1.1 \times 10^{-11}$ to $5.6 \times 10^{-11}$ $\mathrm{S} / \mathrm{cm}$. We have compared these results with variable range hopping theory and find that very few radical sites are electrically active. These results have implications for the production of energy storage systems and motivate future research seeking to increase conductivity in stable radical containing materials.

\section{Supplementary Material}

Refer to Web version on PubMed Central for supplementary material.

\section{Acknowledgments}

This work was supported the Department of Energy Office of Basic Energy Science (Grant DE-SC0014336). We acknowledge use of the facilities of the Cornell NanoScale Facility, a member of the National Nanotechnology Coordinated Infrastructure (NNCI), supported by the National Science Foundation (NSF) (Grant ECCS-1542081). One of us (AC) is grateful to the National Science Foundation for a Graduate Research Fellowship. This material is based upon work supported by the National Science Foundation Graduate Research Fellowship under Grant No. DGE-1650441. We also acknowledge the use of shared facilities of the Cornell Center for Materials Research which is supported through the NSF MRSEC program (Grant DMR-1719875) and the shared facilities of the National Biomedical Center for Advanced Electron Spin Resonance Technology, which is supported by the National Institute of General Medical Sciences, a part of the National Institutes of Health (Grant P41GM103521)

\section{Notes and references}

1. Suga T, Ohshiro H, Ugita S, Oyaizu K, Nishide H. Adv Mater. 2009; 21:1627-1630.

2. Nishide H, Iwasa S, Pu YJ, Suga T, Nakahara K, Satoh M. Electrochim Acta. 2004; 50:827-831.

3. Nishide H, Oyaizu K. Science. 2008; 319:737-738. [PubMed: 18258887]

4. Nishide H, Suga T. Electrochem Soc Interface. 2005; 14:32-36.

5. Nakahara K, Oyaizu K, Nishide H. Chem Lett. 2011; 40:222-227.

6. Janoschka T, Hager MD, Schubert US. Adv Mater. 2012; 24:6397-6409. [PubMed: 23238940]

7. Nishide H. Adv Mater. 1995; 7:937-941.

8. Wenning, B. PhD thesis. Cornell Univerisity; 2016.

9. Hawker CJ, Bosman AW, Harth E. Chem Rev. 2001; 101:3661-3688. [PubMed: 11740918]

10. Kim JK, Cheruvally G, Choi JW, Ahn JH, Lee SH, Choi DS, Song CE. Solid State Ionics. 2007; 178:1546-1551.

11. Nakahara K, Iwasa S, Satoh M, Morioka Y, Iriyama J, Suguro M, Hasegawa E. Chem Phys Lett. 2002; 359:351-354.

12. Kim JK, Kim Y, Park S, Ko H, Kim Y. Energy Environ Sci. 2016; 9:1264-1269.

13. Kim JK, Scheers J, Ahn JH, Johansson P, Matic A, Jacobsson P. J Mater Chem A. 2013; 1:24262430.

14. Liu CM, Chen J, Wang FQ, Yi BL. Russ J Electrochem. 2012; 48:1052-1057.

15. Kim Y, Jo C, Lee J, Lee CW, Yoon S. J Mater Chem. 2012; 22:1453-1458.

16. Guo W, Yin YX, Xin S, Guo YG, Wan LJ. Energy Environ Sci. 2012; 5:5221-5225.

17. Rostro L, Baradwaj AG, Boudouris BW. ACS Appl Mater Interfaces. 2013; 5:9896-9901. [PubMed: 24044350]

18. Baradwaj AG, Rostro L, Boudouris BW. Macromol Chem Phys. 2016; 217:477-484. 
19. Baradwaj AG, Rostro L, Alam MA, Boudouris BW. Appl Phys Lett. 2014; 104:213306.

20. Rostro L, Wong SH, Boudouris BW. Macromolecules. 2014; 47:3713-3719.

21. Baradwaj AG, Wong SH, Laster JS, Wingate AJ, Hay ME, Boudouris BW. Macromolecules. 2016; 49:4784-4791.

22. Karlsson C, Suga T, Nishide H. ACS Appl Mater Interfaces. 2017; 9:10692-10698. [PubMed: 28282111]

23. Oyaizu K, Ando Y, Konishi H, Nishide H. J Am Chem Soc. 2008; 130:14459-14461. [PubMed: 18842045]

24. Oyaizu K, Nishide H. Adv Mater. 2009; 21:2339-2344.

25. Behrends F, Wagner H, Studer A, Niehaus O, Pöttgen R, Eckert H. Macromolecules. 2013; 46:2553-2561.

26. Perrier S, Barner-Kowollik C, Quinn JF, Vana P, Davis TP. Macromolecules. 2002; 35:8300-8306.

27. Wiemann Y, Simmendinger J, Clauss C, Bogani L, Bothner D, Koelle D, Kleiner R, Dressel M, Scheffler M. Appl Phys Lett. 2015; 106:193505.

28. Sukegawa T, Omata H, Masuko I, Oyaizu K, Nishide H. ACS Macro Lett. 2014; 3:240-243.

29. Bugnon L, Morton CJH, Novak P, Vetter J, Nesvadba P. Chem Mater. 2007; 19:2910-2914.

30. Volodarsky, LB., Reznikov, VA., Ovcharenko, VI. Synthetic Chemistry of Stable Nitroxides. CRC Press; Boca Raton: 1993.

31. Kemper TW, Larsen RE, Gennett T. J Phys Chem C. 2014; 118:17213-17220.

32. Suga T, Takeuchi S, Ozaki T, Sakata M, Oyaizu K, Nishide H. Chem Lett. 2009; 38:1160-1161.

33. Lee K, Kim JY, Park SH, Kim SH, Cho S, Heeger AJ. Adv Mater. 2007; 19:2445-2449.

34. Monkman AP, Burrows HD, da Miguel MG, Hamblett I, Navaratnam S. Chem Phys Lett. 1999; 307:303-309.

35. Xing KZ, Johansson N, Beamson G, Clark DT, Bredas J, Salaneck WR. Adv Mater. 1997; 13:1027-1031.

36. Scurlock RD, Wang B, Ogilby PR, Sheats JR, Clough RL. J Am Chem Soc. 1995; 117:1019410202.

37. Davis MS, Mao C, Kreilick RW. Mol Phys. 1975; 29:665-672.

38. Mao CR, Kreilick RW. Chem Phys Lett. 1975; 34:447-450.

39. Gearba IR, Nam CY, Pindak R, Black CT. Appl Phys Lett. 2009; 95:173307.

40. Wang Y, Rubner MF. Synth Met. 1990; 39:153-175.

41. Shklovskii, BI., Efros, AL. Electronic Properties of Doped Semiconductors. Springer; Berlin: 1984.

42. Miller A, Abrahams E. Phys Rev. 1960; 120:745.

43. Mark, JE. Physical Properties of Polymers Handbook. Springer; New York: 2007.

44. Kemper TW, Larsen RE, Gennett T. J Phys Chem C. 2015; 119:21369-21375.

45. Northrup JE. Appl Phys Lett. 2011; 99:062111.

J Mater Chem C Mater. Author manuscript; available in PMC 2019 January 07. 

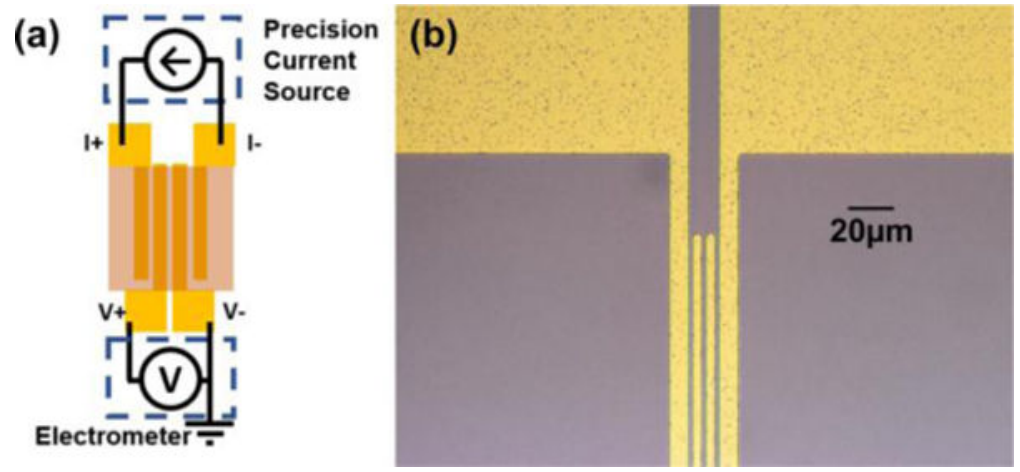

Figure 1.

(a) Schematics of circuit for conductivity measurement. (b) Optical image of fabricated template with $2 \mu \mathrm{m}$ spacing between electrodes. 


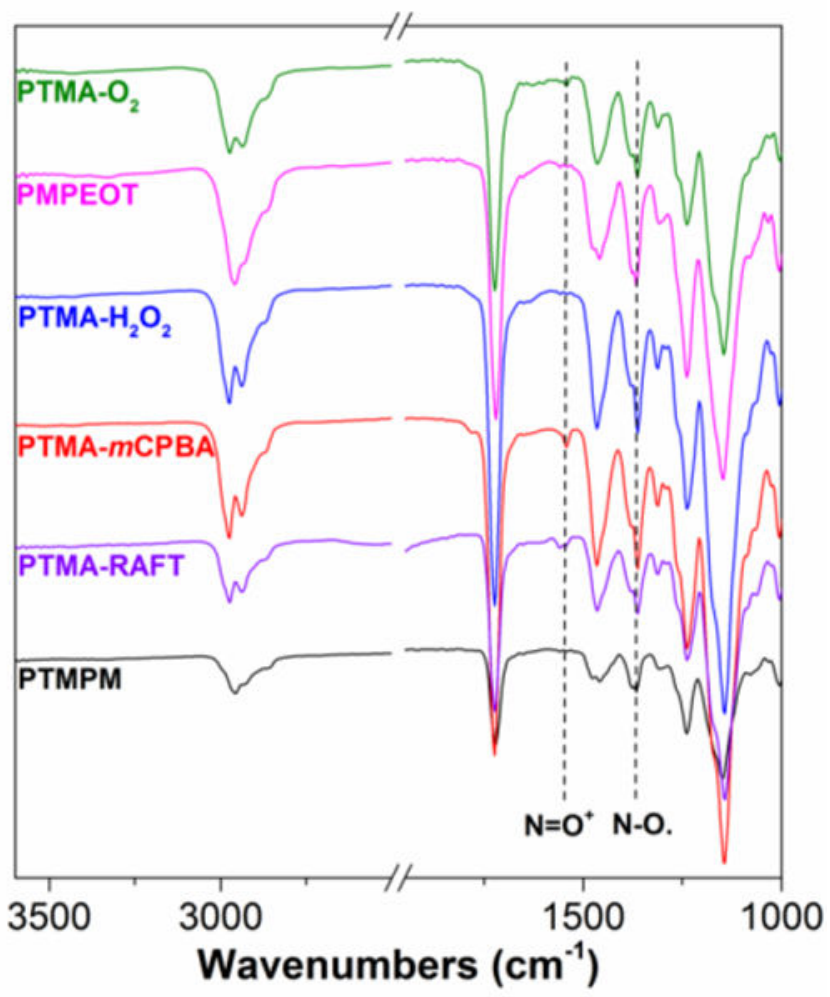

Figure 2.

FTIR of PTMA samples and the precursor polymers. The peak at $1365 \mathrm{~cm}^{-1}$ is associated with $\mathrm{N}-\mathrm{O} \cdot$, while the peak at $1540 \mathrm{~cm}^{-1}$ is associated with ${ }^{+} \mathrm{N}=\mathrm{O}$. The ${ }^{+} \mathrm{N}=\mathrm{O}$ peak is only observable in $m$ CPBA-oxidized PTMA (PTMA-RAFT, PTMA- $m$ CPBA). 

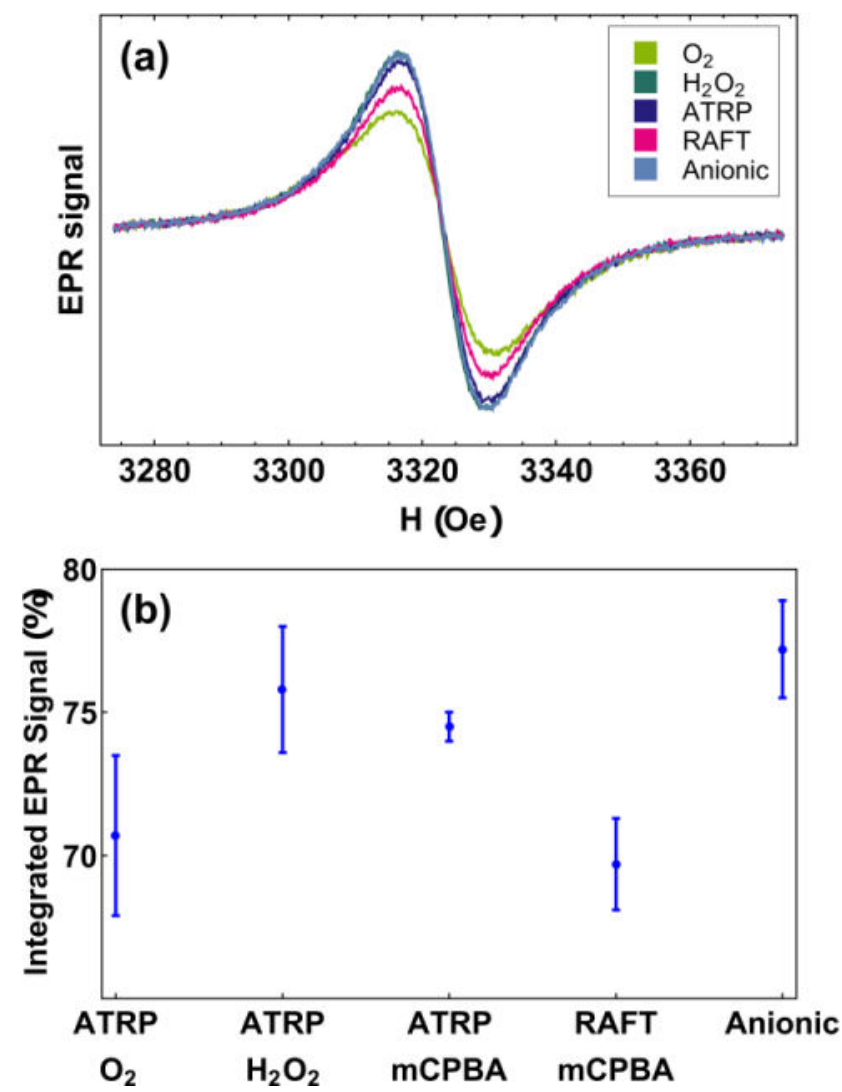

Figure 3.

(a) EPR lineshapes of PTMA samples. (b) Radical yield of PTMA samples determined by liquid-state EPR. 

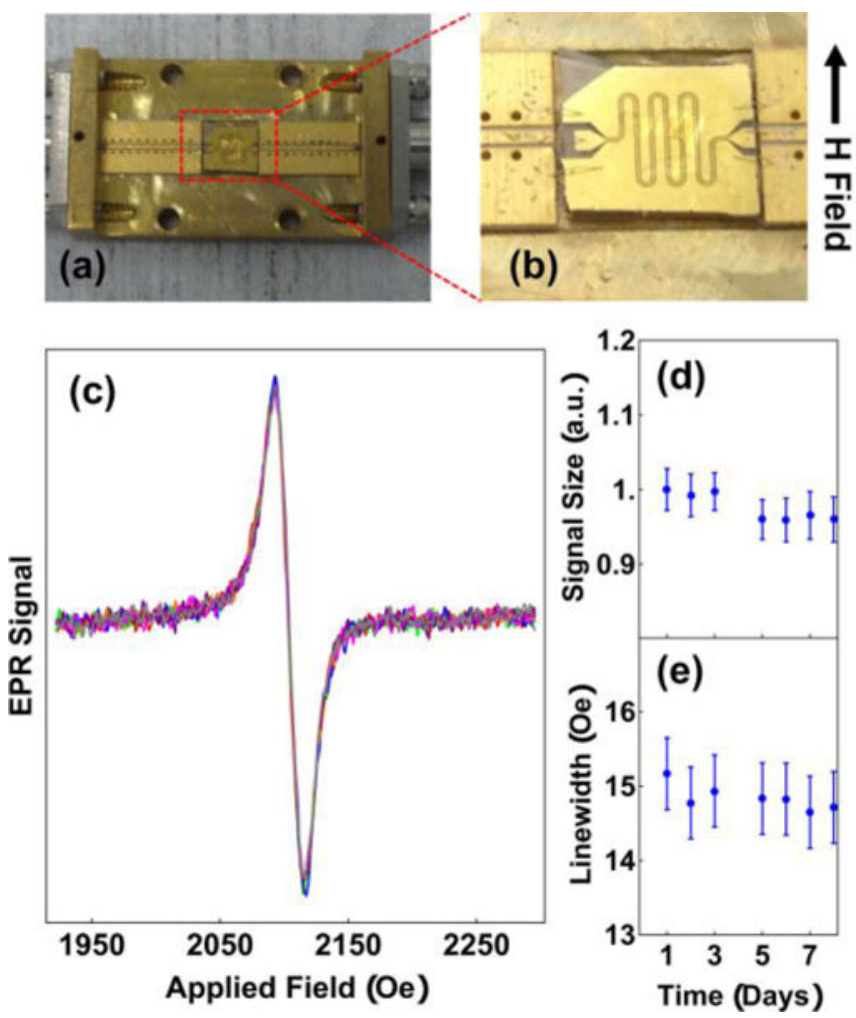

Figure 4.

EPR (a) Brass box with coplanar waveguide for microwave experiments (b) RF waveguide wire bonded to a box with sample drop casted on top. (c) Overlay of on-chip EPR curves taken for 8 days (d-e) Change in size of the signal and linewidth over the course of 8 days [will change to share borders] 


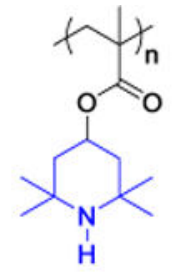

PTMPM

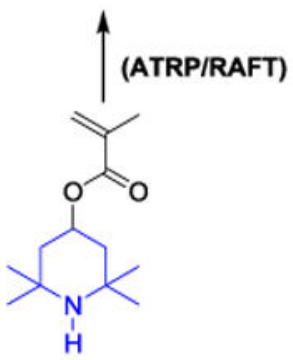

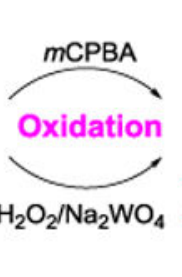

4

PTMA

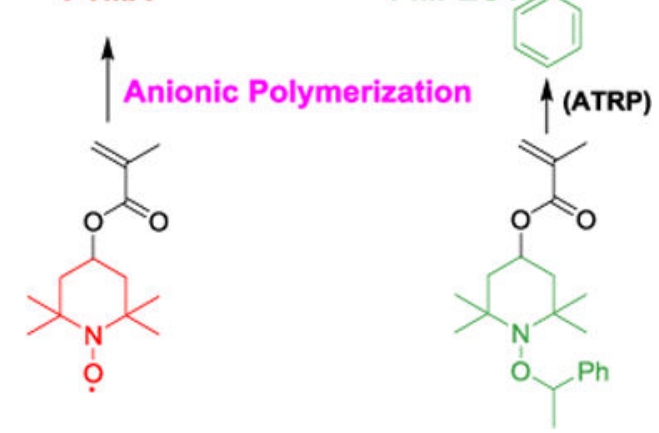

Scheme 1.

Synthesis of PTMA and precursor polymers via living polymerization and subsequent oxidation. 


\section{Table 1}

Preparation methods and GPC data of PTMA

\begin{tabular}{|c|c|c|c|c|}
\hline Sample & Polymerization & Oxidation & $M_{n}(K g / m o l)$ & D \\
\hline PTMA-Anionic & Anionic & - & 19.9 & 1.22 \\
\hline РТМА- $m C$ СВА & ATRP & $m C$ PBA & 24.7 & 1.15 \\
\hline PTMA- $\mathrm{H}_{2} \mathrm{O}_{2}$ & ATRP & $\mathrm{H}_{2} \mathrm{O}_{2}$ & 24.4 & 1.16 \\
\hline PTMA-O ${ }_{2}$ & ATRP & $\mathrm{O}_{2}$ & 14.2 & 1.17 \\
\hline PTMA-RAFT & RAFT & $m \mathrm{CPBA}$ & 17.8 & 1.28 \\
\hline
\end{tabular}




\section{Table 2}

Conductivity of PTMA prepared by different polymerization and oxidation methods

\begin{tabular}{cccc} 
Sample & Polymerization & Oxidation & Conductivity (S/cm) \\
\hline PTMA-Anionic & Anionic & - & $1.09 \pm 0.76 \times 10^{-11}$ \\
PTMA- $\mathrm{H}_{2} \mathrm{O}_{2}$ & ATRP & $\mathrm{H}_{2} \mathrm{O}_{2}$ & $5.59 \pm 0.78 \times 10^{-11}$ \\
PTMA-RAFT & RAFT & $m$ CPBA & $2.54 \pm 0.31 \times 10^{-11}$ \\
P3HT & - & - & $4.46 \pm 2.03 \times 10^{-8}$ \\
\hline
\end{tabular}

